\title{
Evaluation of The Efficacy of Oxytetracycline on Experimentally Induced Caprine Coccidiosis Due to Eimeria arloingi Infection
}

\author{
MikaiL Hudu Garba ${ }^{* 1,2}$, Shehu Na' Allah Alhaji Saidu ${ }^{1}$ and Mohammed Mamman ${ }^{1,3}$ \\ ${ }^{I}$ Medicine Specialty, College of Veterinary Surgeons of Nigeria, Zaria Study Centre, Nigeria \\ ${ }^{2}$ Department of Pharmacology and Toxicology, Faculty of Veterinary Medicine, University of Abuja, Nigeria \\ ${ }^{3}$ Department of Pharmacology and Toxicology, Faculty of Veterinary Medicine, Ahmadu Bello University, Zaria, Nigeria \\ *Corresponding author's Email: mghudu@yahoo.com; (DoRCiD: 0000-0001-5964-6672
}

\begin{abstract}
Coccidiosis is a protozoan disease caused by members of the genus Eimeria that affect domestic animal species. The current study was aimed at evaluating the effect of oxytetracycline administration on experimental caprine coccidiosis. Sixteen red Sokoto goat kids divided into four groups (A to D) of four goat kids each, were used for the study. Groups A, B and C were infected by oral inoculation with two ml containing $1.5 \times 10^{3}$ sporulated oocysts of Eimeria arlongi per animal, while group D was the neutral control group. Group A was treated with $10 \%$ oxytetracycline intramuscularly daily for five days. Group B was treated with Sulfadimidine $33.3 \%$ subcutaneously daily for five days and group $\mathrm{C}$ served as an infected untreated group. Fecal oocysts per gram count was conducted during the experiment. The present result showed a significant decrease $(\mathrm{P} \leq 0.05)$ in fecal oocysts load in the treated groups. Neither schizonts nor merozoites were detected in the intestinal smear of kid treated with oxytetracycline but were detected in the intestinal smear of infected untreated goat kid. Cystic degenerative changes were seen in the intestinal glandular cells of the infected untreated goat kid. Conclusively, the current finding suggests that oxytetracycline can effectively be used in treating caprine coccidiosis.
\end{abstract}

Keywords: Coccidiosis, Caprine, Eimeria arlongi, Goat Kids, Oxytetracycline, Treatment

\section{INTRODUCTION}

Coccidiosis is a protozoan disease caused by members of the genus Eimeria that affect domestic animal species (Geremew, 2018). It is also a disease that causes adverse effects on the general health of various domestic animals. Acute invasion and destruction of the intestinal mucosa, diarrhea, fever, anorexia, emaciation, weight loss and sometimes death are characteristics of the disease (Geremew, 2018). In goats, coccidiosis is an enteric parasitic disease caused by multiple protozoan parasite species of the genus Eimeria (Engidaw et al., 2015). Goats production in recent years is faced with major constraints of high kids' mortalities due to the disease condition (Engidaw et al., 2015). Invasion and destruction of the hosts' intestinal mucosal cells by coccidia could lead to electrolyte loss, poor absorption of nutrients and anemia (Engidaw et al., 2015). Diarrhea is one of the most common clinical symptoms of infection and affected animals can show a rough hair coat, weakness and poor weight gain (Geremew, 2018).

The disease can be diagnosed based on clinical findings such as diarrhea, dehydration and progressive emaciation, and the presence of large numbers of oocysts in the feces (Engidaw et al., 2015) along with a demonstration of the organisms in intestinal mucosal smear during post mortem. Anticoccidial drug therapy either orally or parenterally is the main treatment of coccidiosis (Papich, 2018). The largest serving anticoccidial agents in small animals and poultry are Sulfonamide drugs (Papich, 2018). They include Sulfadimethoxine, Sulfaquinoxaline, and Sulfamerazine, Sulfachloropyrazine, etc (Papich, 2018). Coccidian parasites are developing resistance to common anticoccidial drugs (Papich, 2018). Oxytetracycline has a broad spectrum of activity against protozoan parasites, but there is little or no literature regarding its proven efficacy scientifically against coccidian parasites (Papich, 2018). It was therefore imperative to investigate the effectiveness of oxytetracycline scientifically against coccidian parasites in small ruminants. The aim of the present study was to evaluate the effects of oxytetracycline administration on experimental coccidiosis in goat kids.

\section{MATERIALS AND METHODS}

\section{Experimental animals}

Sixteen kids aged between 4 to 10 weeks were purchased from a market in Zaria Local Government Area of Kaduna state, Nigeria. The animals were screened for the presence of coccidian parasites/Eimeria oocysts using simple 
flotation technique (Geremew, 2018). The kids were allowed to acclimatize for seven days and were fed with maize bran, beans, hay and water ad libitum during the experimental period.

\section{Ethical approval}

The experiment was conducted according to the University of Abuja, Nigeria, Ethical Committee, on Animal Use (UAECAU/2018), along with ethical permission from the Ahmadu Bello University Zaria, Nigeria, committee, on animal use and care (ABUCAUC) with approval number: (ABUCAUC/2020/48).

\section{Isolation and identification of coccidia oocysts}

Animals were diagnosed positively infected using simple flotation technique and light microscopy method of detecting oocysts of Eimeria species (Geremew, 2018). Six grams of feces were collected and filtered through a sieve covered with folded gauze from animals positively infected with Eimeria oocysts (Rakhshandehroo et al., 2013). The material filtered was centrifuged for 10 minutes at $250 \mathrm{~g}$. The supernatant was then removed. Oocysts sporulation was achieved by using $2.5 \%$ (w/v) potassium dichromate solution as described by Rakhshandehroo et al. (2014). Identification of the oocysts specie was done as reported by Rakhshandehroo et al. (2014).

\section{Experimental design}

The sixteen kids were divided into four groups (A to D) of four animals each. Groups A, B and C were infected by oral inoculation with two $\mathrm{ml}$ containing $1.5 \times 10^{3}$ sporulated oocysts per animal, while group D remained as uninfected untreated neutral control group. Group A kids were treated with $10 \%$ oxytetracycline (OCECURE®, India) intramuscularly daily for five days at the dose rate of $10 \mathrm{mg} / \mathrm{kg}$. Group B kids were treated with the common anticoccidial agent, sulfadimidine 33.3\% (Shanghai Gongyi, China) subcutaneously daily for five days at the dose rate of $30 \mathrm{mg} / \mathrm{kg}$ and Group C served as infected untreated negative control group. All treatments were initiated on the day after the oocysts were first noticed in the infected kids' feces by microscopy.

\section{Determination of fecal oocysts load}

Fecal samples were collected from each kid in all the groups and examined for the presence of oocysts, using the sedimentation-floatation technique in a sucrose saturated solution (El-Ashram and Suo, 2017). Oocysts Per Gram (OPG) of feces counts were conducted using the McMaster technique (Kheirandish et al., 2014). Calculation of OPG was performed on days 5, 7, 9, and 14 Days' Post Inoculation (DPI) and thereafter on daily basis during the treatment regimen on days 16,17,18,19 and 20 and on days 21 to 27, the post-treatment days, up to the end of the experiment on day 28 to establish decrease or absence of Eimeria oocysts in the feces of the experimental animals.

\section{Intestinal smears and histopathological evaluations}

At the end of the experiment, a kid from each group was sacrificed humanely, intestinal mucosal smears were taken and stained with Giemsa stain and examined under oil immersion micoscopy for viewing the schizonts and merozoites at $\times 1000$ magnification. For histopathological evaluation, $2 \mathrm{~cm}^{2}$ tissue pieces from the caeca and posterior region of the small intestine were collected and fixed in $10 \%$ buffered formalin solution. Multiple transverse slices were embedded in paraffin wax. Sections were cut at $5 \mu \mathrm{m}$, and all were stained with hematoxylin-eosin (Gelberg, 2012).

\section{Data management and analysis}

Data obtained from the study were presented in tables, figures and plates. Results were presented as mean plus/minus standard error of the mean (Mean \pm SEM). The data were also analyzed using one-way analysis of variance (ANOVA) followed by Tukey post-hoc with the aid of SPSS statistical software (version 4.0). Values $\leq 0.05$ were considered statistically significant.

\section{RESULTS}

\section{Oocysts counts in the experimentally infected kids}

Coccidia oocysts were detected in the feces of the kids inoculated with E. arloingi (Figure 1). A significant decrease $(\mathrm{P} \leq 0.05)$ was recorded on days 18 and 19 on fecal OPG counts in the groups treated with oxytetracycline and sulfadimidine when compared with the infected untreated group (Figure 2), as well as on days 22 to 28 (Figure 3). Treatment with oxytetracycline eliminated the coccidian oocysts on day 19 in one of the kids in group A, sulfadimidine treatment eliminated the coccidian oocysts on day 22 in one of the kids in group B, while fecal oocyst per gram counts of 14,440 (OPG) was recorded in one of the infected untreated kids in group $\mathrm{C}$ on day 28, which was the last day of the experiment (Figure 4). Neither schizonts nor merozoites were detected in the intestinal smear of kid treated with oxytetracycline on day 28. However, at the end of the experiment (day 28), schizonts were detected in the intestinal 
smear of kid treated with sulfadimidine and both schizonts and merozoites were detected in the intestinal smear of the infected untreated kid (Table 1 and figure 5).

Effect of treatment with oxytetracycline on the intestinal histopathology of kids experimentally infected with $E$. arloingi

There was no histopathological lesion seen in the group treated with oxytetracycline (Figure 6). Cystic degenerative changes were seen in intestinal glandular cells of the infected untreated group (Figure 7). Moreover, no histopathological lesion was seen in the uninfected untreated group (Figure 8).

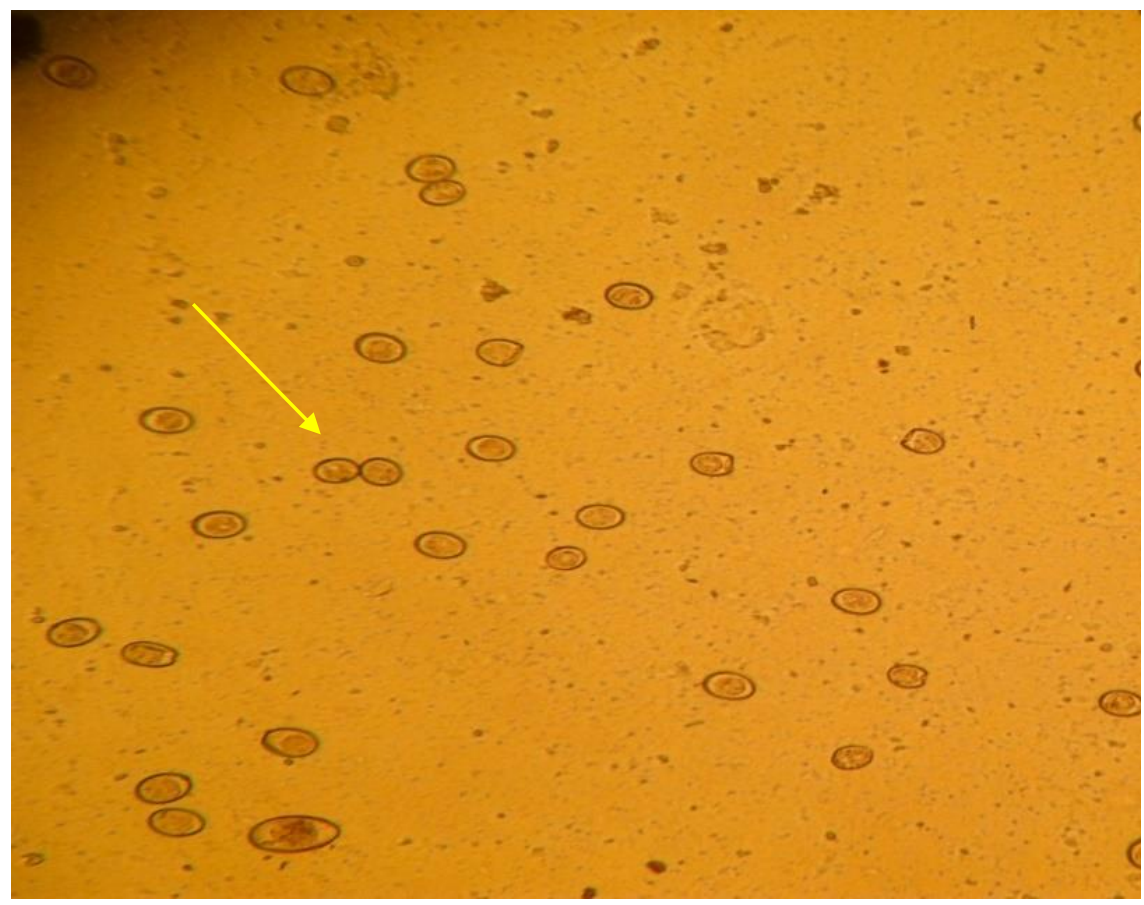

Figure 1. Microscopic view of oocysts (arrow) from fecal sample of experimentally infected kid with Eimeria arloingi oocysts in Ahmadu Bello University (A.B.U.) Zaria, Nigeria on June 2018 (× 100 magnification)

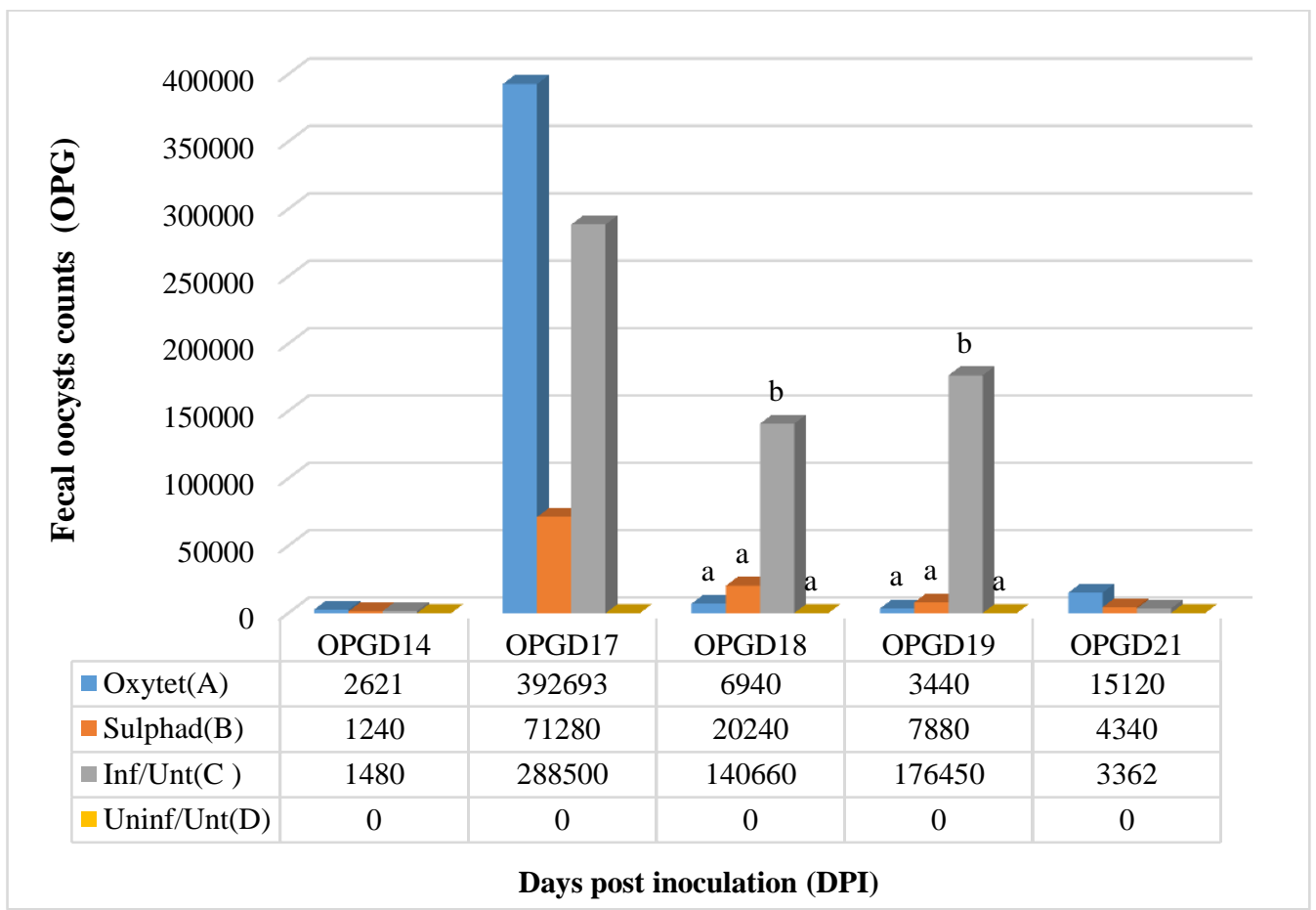

Figure 2. Effect of oxytetracycline treatment on fecal oocysts count in experimental caprine coccidiosis of kids infected with Eimeria arloingi oocysts, 14 - 21 days' post inoculation in A.B.U. Zaria, Nigeria on June 2018. Data are presented as mean \pm SEM. Group A: Infected and treated with 10\%; oxytetracycline; Group B: Infected and treated with sulphadimidine; Group C: Infected and untreated; Group D: Uninfected and untreated; a-b: Significant difference (P $\leq$ 0.05 ) between group C and groups A, B and D; OPG: Oocysts per gram; DPI: Days post inoculation; D: Day; Oxytet: Oxytetracycline; Sulphad : Sulphadimidine 


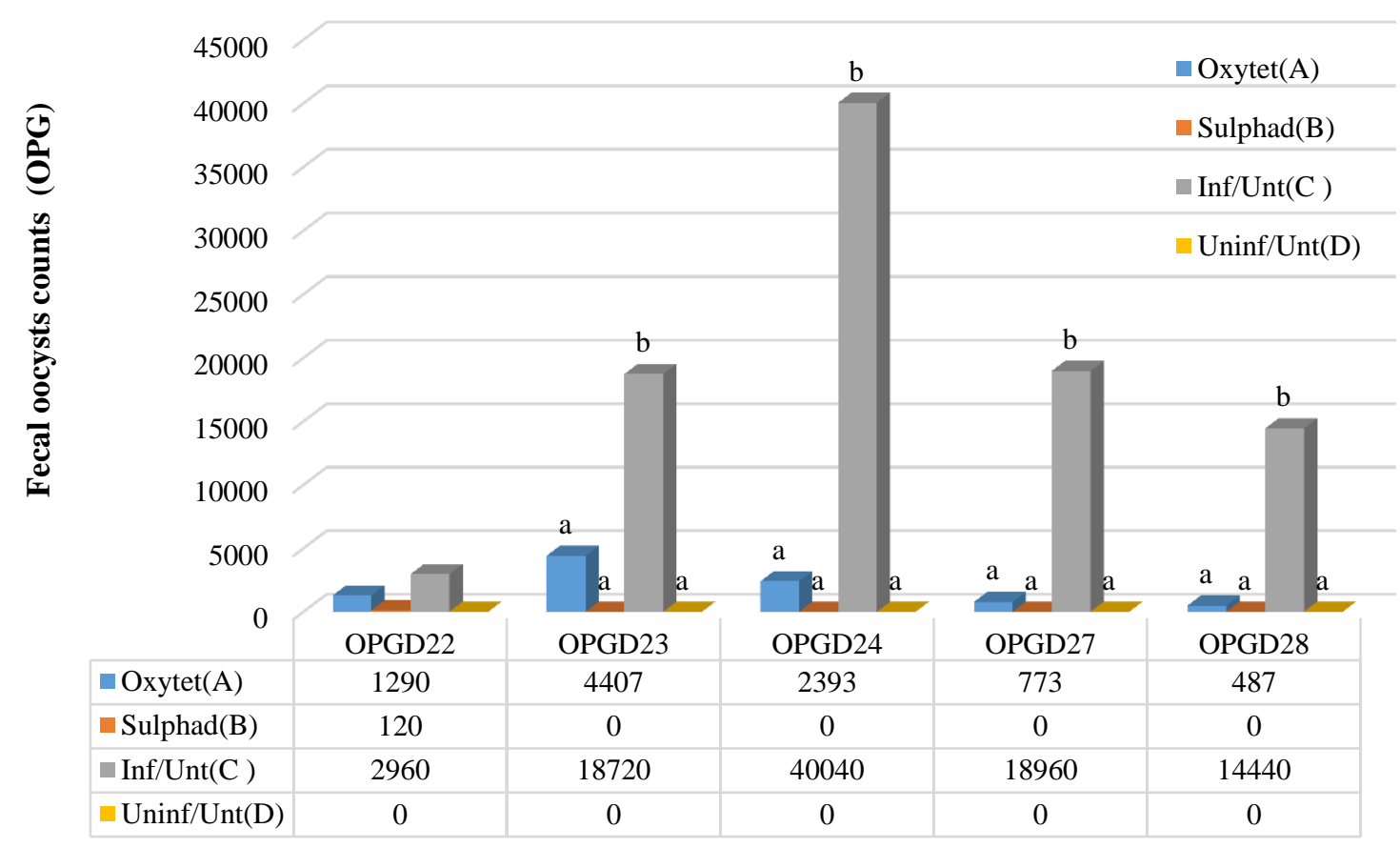

Days post inoculation (DPI)

Figure 3. Effect of oxytetracycline treatment on fecal oocysts count in experimental caprine coccidiosis of kids infected with Eimeria arloingi oocysts, 22 - 28 days' post inoculation in A.B.U. Zaria, Nigeria on June 2018. Data are presented as mean \pm SEM; a-b: Significant difference $(\mathrm{P} \leq 0.05)$ between group $\mathrm{C}$ and groups A, B and D; Group A: Infected and treated with $10 \%$ oxytetracycline; Group; B: Infected and treated with sulphadimidine; Group C: Infected and untreated; Group D: Uninfected and untreated; OPG: Oocysts per gram; DPI: Days post inoculation; D: Day; Oxytet: Oxytetracycline; Sulphad: Sulphadimidine

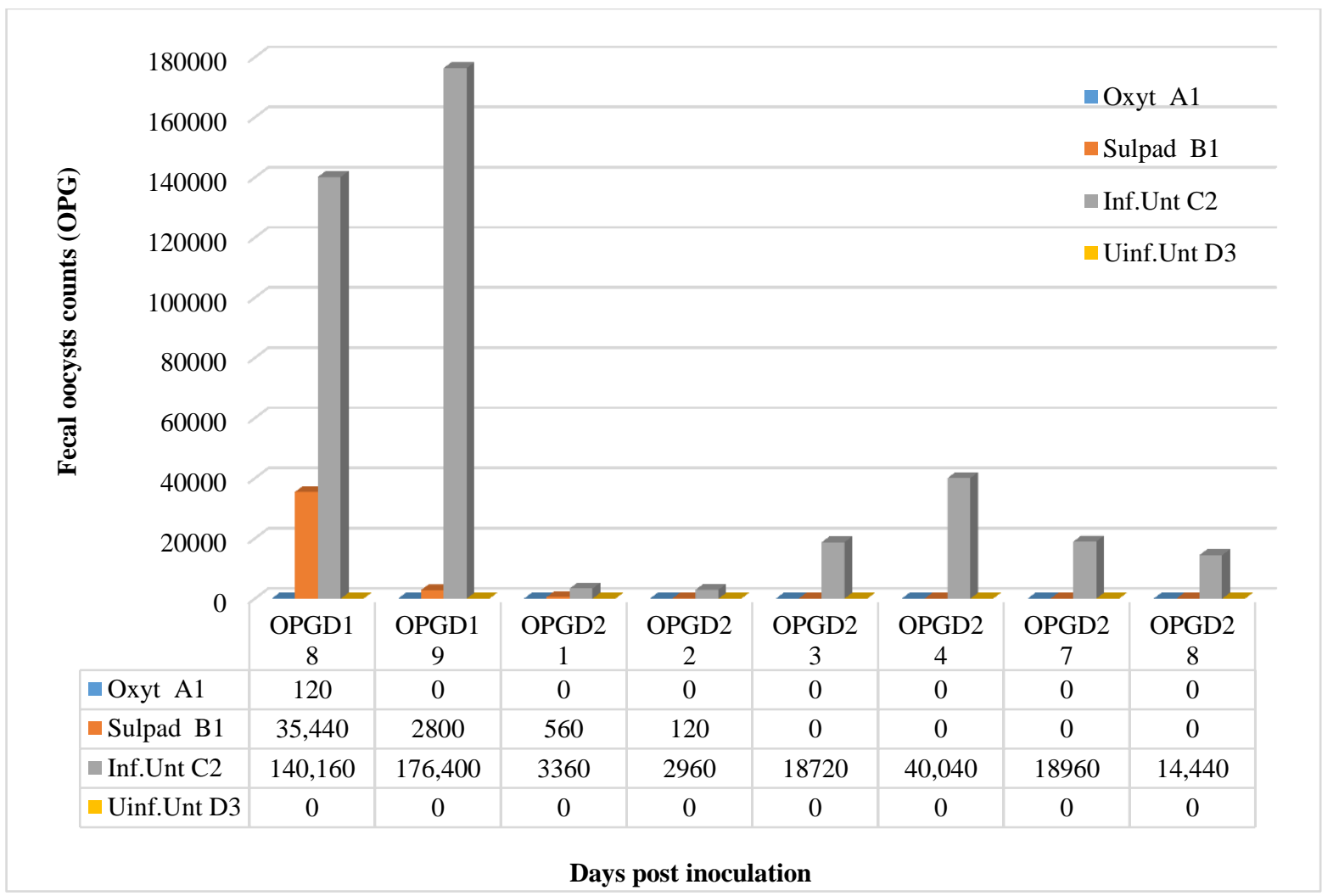

Figure 4. Effect of oxytetracycline treatment on fecal oocysts count in experimental caprine coccidiosis of kids per group infected with Eimeria arloingi oocysts, 18 - 28 days' post inoculation in A.B.U. Zaria, Nigeria on June 2018. Group A: Infected and treated with $10 \%$ oxytetracycline; Group B: Infected and treated with sulphadimidine; Group C: Infected and untreated; Group D: Uninfected and untreated; OPG: Oocysts per gram; DPI: Days post inoculation; D: Day; Oxytet: Oxytetracycline; Sulphad: Sulphadimidine 

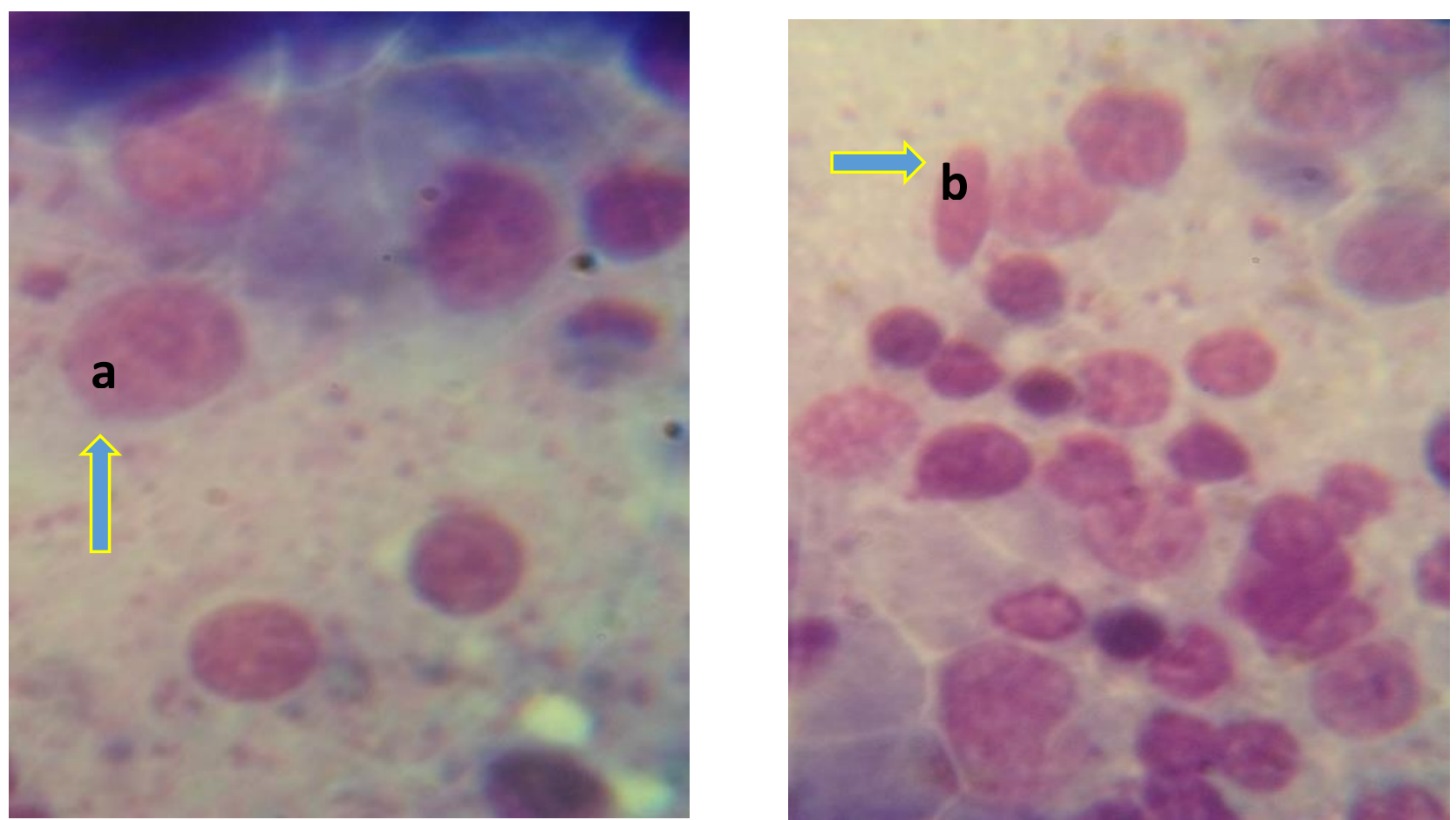

Figure 5. Microscopic view of schizonts (a) and merozoites (b) (Oil immersion $\times 1000$ magnification) in the intestinal smear of infected untreated kid in A.B.U. Zaria, Nigeria on June 2018.

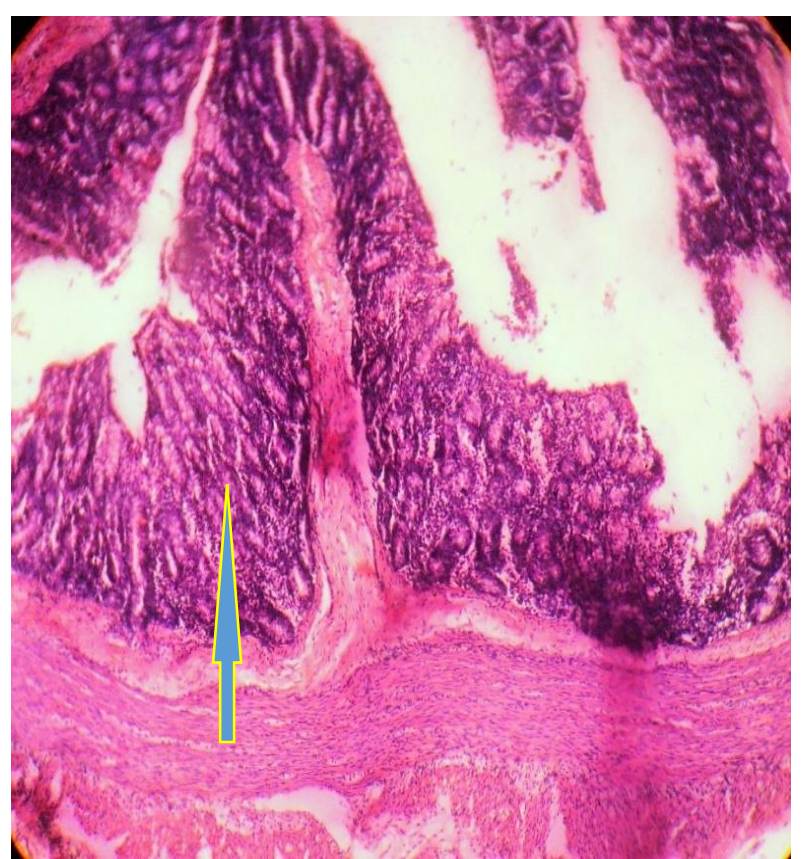

Figure 6. Histopathological findings from the intestine of a kid infected experimentally with Eimeria arloingi oocysts and treated with $10 \%$ oxytetracycline in A.B.U. Zaria, Nigeria on June 2018 (group A) showing intact glandular cells (arrow) $\mathrm{H}$ and $\mathrm{E} \times 200$

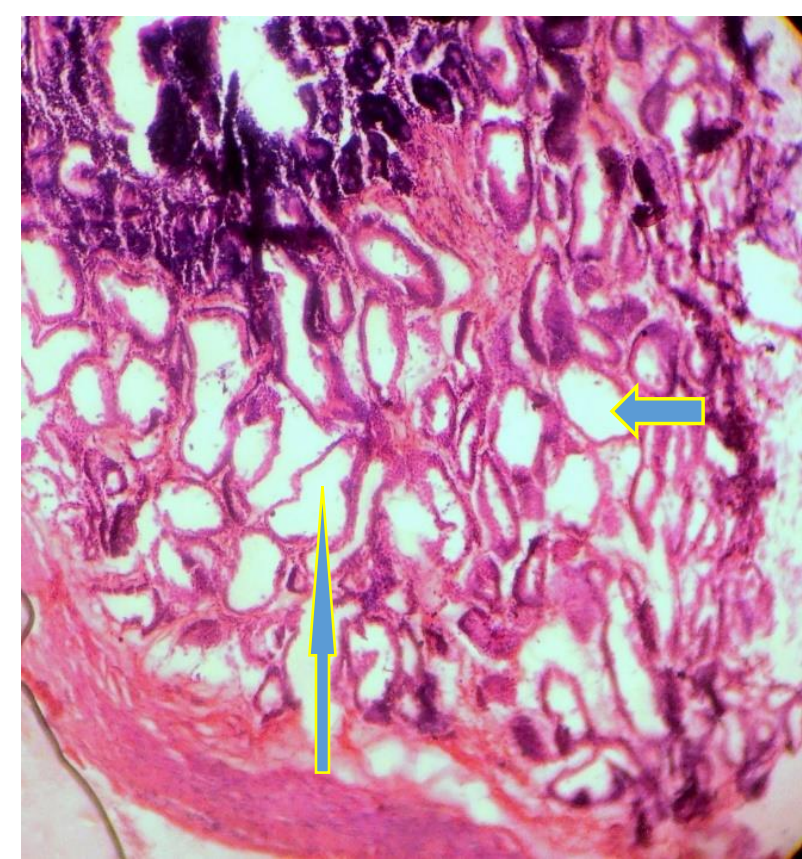

Figure 7. Histopathological findings from the intestine of kid experimentally infected with Eimeria arloingi oocysts but not treated in A.B.U. Zaria, Nigeria on June 2018 showing cystic degenerative changes (arrow) $\mathrm{H}$ and $\mathrm{E} \times 200$

Table 1. Findings of the kids' intestinal smear after experimental infection with Eimeria arloingi oocysts and treatment with antibiotics in A.B.U. Zaria, Nigeria on June 2018

\begin{tabular}{lccc}
\hline Experimental groups & \multirow{2}{*}{ Animals sacrificed } & \multicolumn{2}{c}{ Intestinal regions } \\
\cline { 3 - 4 } A (10\% Oxytetracycline) & A1 & Caecum & Ileum \\
B (Sulphadimidine) & B1 & Schizonts & $-\mathrm{ve}$ \\
C (Infected untreated) & C2 & Schizonts & Schizonts \\
D (Uninfected untreated) & D4 & Merozoites & $-\mathrm{ve}$ \\
\hline -ve: Absence of schizont or merozoite & & $-\mathrm{ve}$ &
\end{tabular}




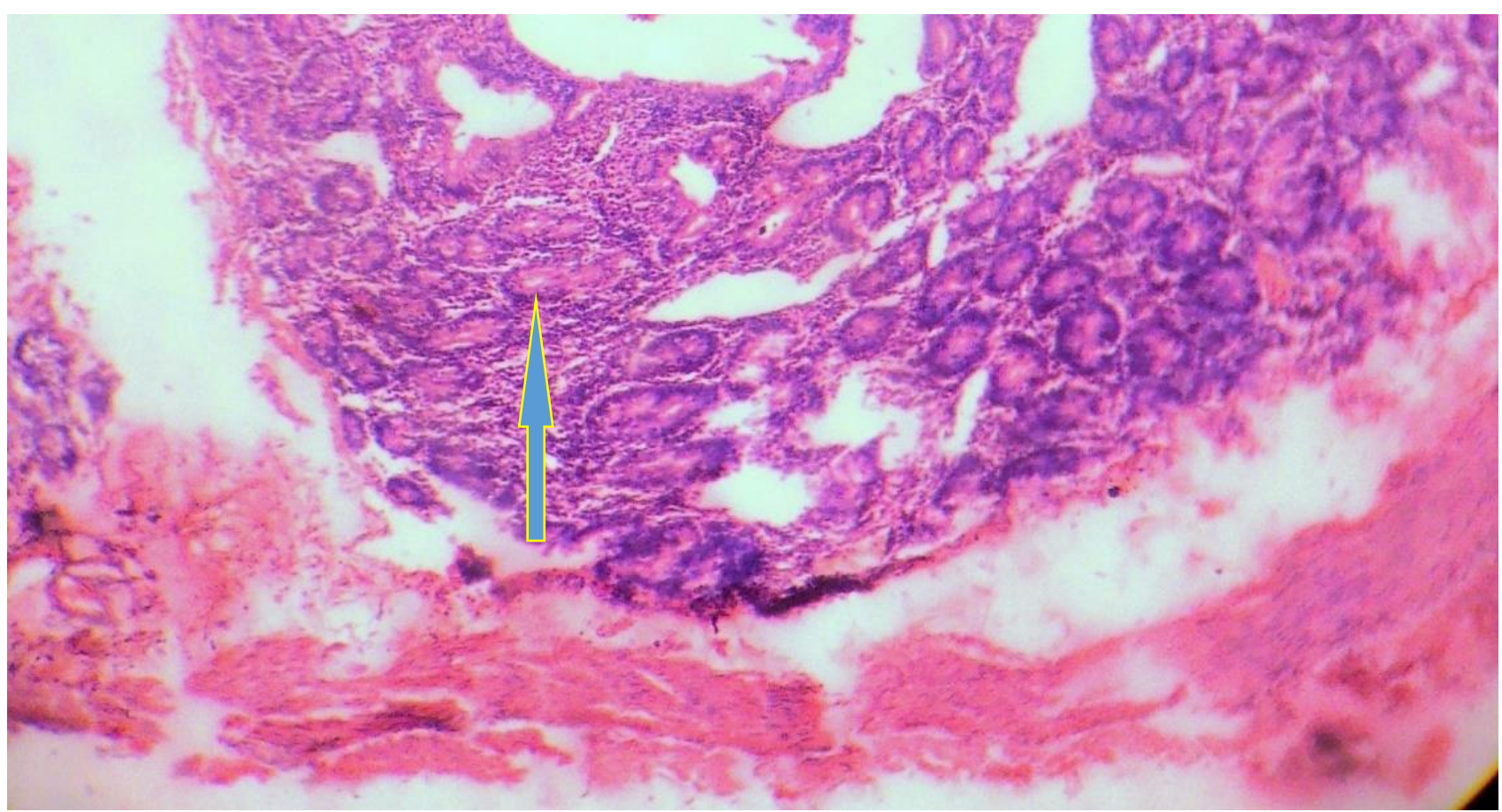

Figure 8. Histopathological findings from the intestine of uninfected untreated kid in A.B.U. Zaria, Nigeria on June 2018 showing an intact intestinal glandular cell (arrow) and $\mathrm{E} \times 200$

\section{DISCUSSION}

Diarrhea is one of the symptoms of coccidiosis and may be bloody in some cases. Coccidian oocyst shed in the feces of carrier animals that did not reveal symptoms as well as affected animals expressing symptoms of the disease (Richards et al., 2016). The findings of the present study indicated that the kids started shedding the coccidian oocysts at day 14 post inoculation. This finding agrees with the earlier report of Rakhshandehroo et al. (2013) that indicated oocysts shedding could start at days 13 to 14 post inoculation. However, some researchers reported the shedding of E. arloingi oocysts by infected kids at days 16 to 18 post inoculation (Hashemnia et al., 2012).

Animals affected by coccidian parasite show specifically dehydration and weight loss during the period of diarrhea (Khodakaram-Tafti and Hashemnia, 2017). The current study demonstrated that clinical coccidiosis is associated with diarrhea and shedding of Eimeria oocysts in the feces of infected animals.

Damage to the epithelial cells with a subsequent reduction in the number of oocysts is reflected histologically by villous atrophy, crypt hyperplasia and cellular infiltration (Khodakaram-Tafti and Hashemnia, 2017). The present study revealed that treatment with oxytetracycline has ceased the development and multiplication of coccidian parasites and protected the glandular cells of the intestine from being damaged by the developmental stages of E. arloingi oocysts (schizonts and merozoites). However, severe damages to the intestinal glandular cells of the infected untreated negative control group were seen due to cystic degenerative changes that took place therein.

\section{CONCLUSION}

The data obtained from the present study revealed that both oxytetracycline and sulfadimidine are effective in reducing and/or clearing fecal oocysts load in the infected animals however oxytetracycline was more effective in stopping the developmental stages of the Eimeria parasites. In conclusion, the current finding suggests that oxytetracycline can be effectively used in treating coccidiosis in small ruminants and particularly in goats.

\section{DECLARATIONS}

\section{Acknowledgement}

We will like to acknowledge the effort of Professors M.Y. Fatihu and M. Bisalla of the Department of Veterinary Pathology, Ahmadu Bello University, Zaria, Nigeria toward the success of this work. Our appreciation also goes to Drs Samaila Danbirni and Grace Uko James and to all the technologists that contributed to the success of this work especially Messrs. Muhammad Yusuf and Hussaini Garba of the Parasitology and Entomology Department, Ahmadu Bello University, Zaria, Nigeria. 


\section{Competing interests}

The authors declared no competing interest exist.

\section{Consent to publish}

All authors were aware and agreed to the fact that the manuscript be published in this journal. This study was not published elsewhere partially or totally.

\section{Authors' contribution}

Mikail HG, Saidu SNA and Mamman M designed the work. Mikail HG conducted the experiment and wrote the manuscript, while Saidu SNA and Mamman M reviewed the manuscript. All authors confirmed the final form of the article.

\section{REFERENCES}

El-Ashram S and Suo X (2017). Electrical cream separator coupled with vacuum filtration for the purification of eimerian oocysts and trichostrongylid eggs. Scientific Reports, 7:43346. DOI: https://doi.org/10.1038/srep43346.

Engidaw, S, Anteneh M, C and Demis C (2015). Coccidiosis in Small Ruminants. African Journal of Basic and Applied Sciences, 7 (6): 311-319. DOI: https://doi.org/10.5829/idosi.ajbas.2015.7.6.96202.

Gelberg HB (2012). Alimentary system and the peritoneum, omentum, mesentery, and peritoneal cavity. In: Zachary JF, McGavin MD, eds. Pathologic basis of veterinary disease. 5th edition. St. Louis, Missouri: Elsevier Mosby Inc, pp. 396-397.

Geremew H (2018). Review on Eimeria: The Role of Characteristic Lesions as Routine Diagnostic Technique. International Journal of Current Research and Academic Review, 6(7): 9-18. DOI: https://doi.org/10.20546/ijcrar.2018.607.002.

Hashemnia M, Khodakaram-Tafti A, Razavi SM and Nazifi S (2012). Experimental caprine coccidiosis caused by Eimeria arloingi: Morphopathologic and electron microscopic studies. Veterinary Research Communications, 36: 47-55. DOI: https://doi.org/10.1007/s11259-011-9511-9.

Kheirandish R, Nourollahi-Fard SR and Yadegari Z (2014). Prevalence and pathology of coccidiosis in goats in southeastern Iran. Journal of Parasitic Diseases, 38(1): 27-31. DOI: https://doi.org/10.1007/s12639-012-0186-0.

Khodakaram-Tafti A and Hashemnia M (2017). An overview of intestinal coccidiosis in sheep and goats. Revue de Médecine Vétérinaire, 167(1-2): 9-20. Available at: https://www.revmedvet.com/2017/RMV168_9_20.pdf

Papich MG (2018). Sulfonamides and potentiated sulfonamides. In Papich MG, Riviere JE, eds. Veterinary Pharmacology and Therapeutics $10^{\text {th }}$ edition. Iowa, USA: Wiley -BalckWell, pp. 796-825.

Rakhshandehroo E, Nazifi S, Razavi SM, Ghane M and Alavi AM (2013). Caprine coccidiosis: the effects of induced infection on certain blood parameters. Veterinarski Arhiv, 83 (6): 623-631. Available at: https://pdfs.semanticscholar.org/d65e/81dc1990f511656ea14086a4e9c4e19b05bf.pdf.

Rakhshandehroo E, Razavi SM and Nazifi S (2014). Experimental caprine coccidiosis: the pattern of changes in antioxidant micronutrients and vitamins. Veterinary Science Development, 4:5533. DOI: https://doi.org/doi.org/10.4081/vsd.2014.5533.

Richards C, Step DL and Giedt EJ (2016). Coccidiosis Treatment and Prevention in Cattle. Oklahoma State University. Oklahoma $\begin{array}{lllll}\text { Cooperative } & \text { Extension } & \text { Fact } & \text { Sheets. Available }\end{array}$ state.edu/dspace/bitstream/handle/2097/39022/JorgeSimroth2018.pdf?sequence=1\&isAllowed=y 